\title{
Vascular dementia classification based on hilbert huang transform as feature extractor
}

\author{
Wan Siti Nur Shafiqa Wan Musa ${ }^{1}$, Mohd Ibrahim Shapiai ${ }^{2}$, Hilman Fauzi ${ }^{3}$, Aznida Firzah Abdul Aziz ${ }^{4}$ \\ ${ }^{1,2,3}$ Department of Electronic System Engineering, Malaysia-Japan International Institute of Technology, Malaysia \\ ${ }^{1,2,3}$ Centre for Artificial Intelligence and Robotics, Universiti Teknologi Malaysia, Malaysia \\ ${ }^{4}$ Department of Family Medicine UKM Medical Centre, Universiti Kebangsaan Malaysia, Malaysia
}

\begin{tabular}{l} 
Article Info \\
\hline Article history: \\
Received Jun 3, 2019 \\
Revised Aug 6, 2019 \\
Accepted Aug 25, 2019 \\
\hline
\end{tabular}

\section{Keywords:}

Electroencephalogram Hilbert huang transform Vascular dementia

\begin{abstract}
Impairment of cognitive and working memory after stroke was common. Vascular dementia (VaD) was a prevalent type of dementia that was caused by an impaired blood supply to the brain because of a series of small strokes. Electroencephalogram (EEG) gives information about brain status and activity, so it had a lot of potential to be used in diagnosing people with dementia. Since the EEG signal is extremely non-linear and non-stationary data, traditional Fourier analysis such as Fast Fourier Transform (FFT) that broadens sinusoidal signals cannot describe the amplitude contribution of each frequency value in specific time. Meanwhile, Hilbert Huang Transform (HHT) was based on the characteristic local time scale of the signal, it can efficiently obtain instantaneous frequency and instantaneous amplitude for nonstationary and nonlinear data. In this paper, HHT was employed as feature extraction method to extract the energy features of frequency bands from post stroke patients and healthy subjects. The extracted features were fed into extreme learning machine (ELM) for classifying post stroke patient with $\mathrm{VaD}$ and healthy subjects. The results of classification accuracy using HHT as feature extractor and FFT as feature extractor were compared. The mean accuracy of classification using HHT was 59.14\%, respectively, while mean accuracy of classification using FFT was $94.4 \%$, respectively, in classifying post stroke patient with $\mathrm{VaD}$ and healthy subjects.
\end{abstract}

Copyright @ 2020 Institute of Advanced Engineering and Science. All rights reserved.

\section{Corresponding Author:}

Mohd Ibrahim Shapiai,

Department of Electronic System Engineeering,

Malaysia-Japan International Institute of Technology,

Centre of Artificial Intelligence and Robotics,

Universiti Teknologi Malaysia, Kuala Lumpur, Malaysia.

Email: md_ibrahim83@utm.my

\section{INTRODUCTION}

Stroke is regarded as the leading risk factor for cerebrovascular disease which affect severe physical disability and cognitive impairment [1]. About 30 percent of people with stroke, experience dementia in the first year of stroke diagnosis [2]. The precondition for $\mathrm{VaD}$ diagnosis is the dysfunction of post-stroke brain memory [3]. Following Alzheimer's disease (AD), $\mathrm{VaD}$ is the second most frequent type of dementia and considered resulting from cerebral vascular disease and ischemic or hemorrhagic brain injury-related [4]. Between 1 and $4 \%$ of elderly people aged 65 years tend to suffer vascular dementia and prevalently doubled 5 to 10 years after this age [5].

Due to post stroke, working memory (WM) is considerably impacted in this condition. WM provides temporary storage and manipulates information within a short time for complex cognitive functions such as attention, comprehension, reasoning, planning and learning [6]. An individual's WM ability can momentarily store about $7 \pm 2$ items $[3,6]$. 
Neuroimaging and non-neuroimaging methods have been used to diagnose stroke. These methods play a significant part in the diagnosis of stroke disease. Magnetic Resonance Imaging (MRI), Positron Emission Tomography (PET), and Single Photon Emission Computed Tomography (SPECT) are neuroimaging methods that widely known used for stroke diagnosis. Although neuroimaging methods are commonly used for stroke diagnosis, they still have several disadvantages [7]. The prevalent disadvantages of the neuroimaging method include high price, time consuming, and radiation risk imposition. In addition to these methods discussed, Electroencephalogram (EEG) is one of the helpful instruments for detecting and tracking impacted brain after stroke dementia. This study describes a method of evaluating EEG signals of post-stroke patient working memory compared to healthy subjects.

EEG has been commonly used in clinical diagnosis because of its high temporal resolution and has been recognized as an efficient physiological technique that represents the hidden cortical abnormalities by offering a measurable understanding for diagnosing or evaluating future dementia severity [5]. The abnormalities in the EEG spectrum of dementia patients such as the slowing of EEG signal activity in dementia patient can be recognize by using spectral analysis [5]. These changes shown in the power spectrum changes in low frequency, in which low frequency power was increased and high frequency power was decreased [8].

For the study of EEG signals, many signal analysis and processing methods have recently been suggested [9-14]. Traditional Fourier spectral analysis was used among these methods to extract EEG signals for dementia detection [10]. The Fourier analysis method such as FFT in which broadens sinusoidal signals cannot describe the amplitude contribution of each frequency value in specific time [9]. Despite the fact that, the EEG is an extremely non-linear and non-stationary process, therefore the Fourier analysis cannot precisely represent the frequency part of the signal. To tackle this issue, some time-frequency analysis techniques have been suggested and used to obtain EEG features [11]. The short time Fourier transform extracts several frames of the signal to be analyzed with a window that moves with time and used to convert EEG fragments into EEG spectrogram representations in time-frequency domain [15]. The wavelet analysis is comparable to the short-term Fourier Transform which used a variable window size across the entire EEG signal length [16]. In fact, the above described techniques of time-frequency analysis have its own advantage in non-stationary signal processing, but they are all based on Fourier method and have not given a unique definition of frequency mathematically.

HHT presents nonlinear frequency correlation or time-spatial characteristics of signal analysis, such as in ECG and EEG. Besides, HHT is an adaptive and intuitive method which help analyzing the nonstationary signals to locate the local changes and providing the ability for time-frequency analysis and the adequacy for complex data [15]. Therefore, in this paper, HHT which is designed specifically for analyzing non-linear and non-stationary data such EEG signal was used as feature extraction method to extract the energy features of frequency bands from post stroke patients and healthy subjects.

\section{RELATED WORKS}

There are different techniques for extracting brain data, such as spatial filtering [17], temporal information [18], spectral information and event-related potential (ERP) [19]. Indeed, instead of using functional magnetic resonance imaging (fMRI), other researcher used spectral analysis as an alternative method [20]. In related study, EEG analysis using spectral-based analysis has been commonly used by late researcher until the recent now. Spectral analysis method often been used by other researcher and this method provides different opinions in different fields of research and offers useful information.

\subsection{Relative Power Ratio}

Relative Power Ratio (RPR) is used to quantify the EEG signal changes in dementia patients in which low frequency is increase in the power spectrum and high frequency is decrease in the power spectrum [21]. The RP for each chosen frequency band was computed using (1) [17].

$$
\mathrm{RP}(\%)=\frac{\sum \text { Selected frequency range }}{\sum \text { Total range }(0.5-64 \mathrm{~Hz})}
$$

\subsection{Spectral Entropy}

Previous studies investigated the EEG signal pattern of stroke patients cognitive and thinking ability. Spectral Entropy (SpecEn) is used to evaluate signal spectrum flatness and is regarded a helpful way to quantify frequency slowdown due to dementia[16]. SpecEn is calculated to apply the Shannon's entropy to the normalized Power Spectral Density (PSD) as shown in [22]. 
SpecEn $=\frac{-1}{\log N} \sum_{\mathrm{f}=0.5 \mathrm{~Hz}}^{64 \mathrm{~Hz}} \log \left[\mathrm{PSD}_{n}(\mathrm{f})\right]$

\subsection{Fourier Analysis}

Using spectral analysis is the common method of assessing EEG. Power spectral shows the EEG signal frequency content or the frequency power distribution. Power spectrum is really helpful for distinguishing activity in the EEG signal [23]. FFT is used to calculate the global EEG value by using Power Spectral Density [24].

\section{METHODOLOGY}

This part addresses this research's experimental procedure from data acquisition to results assessment. Figure 1 showing the research's workflow to analyze stroke patient processed data.

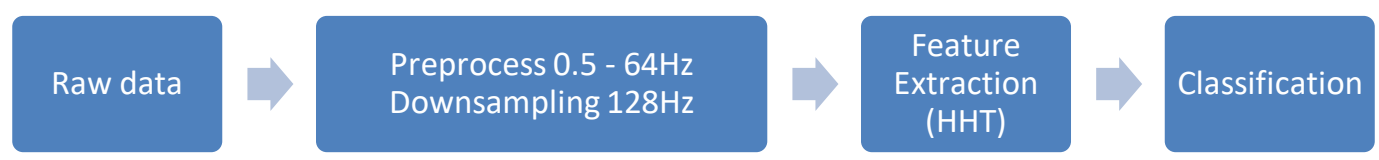

Figure 1. Block diagram of the proposed framework

\subsection{Inclusion and Exclusion Parameter}

EEG data sets for 35 healthy subjects were recorded, age range 52 years, and 50 post stroke patients age ranged 62 years who have severe post stroke after 6 months on set. The patients were recruited from Pusat Perubatan Universiti Kebangsaan Malaysia (PPUKM). The ethical standard of the HUKM Committee on Institutional and Research Ethics complied with all processes conducted in this study involving human respondents. For this study, it took official permission from patients to continue with the EEG data collection by filling out the consent form. As shown in Figure 2, EEG data was recorded using BR32i with a total of 32 electrodes, plus system reference electrodes and the ground with sampling rate of $256 \mathrm{~Hz}$ and $50 \mathrm{~Hz}$ notch filter.

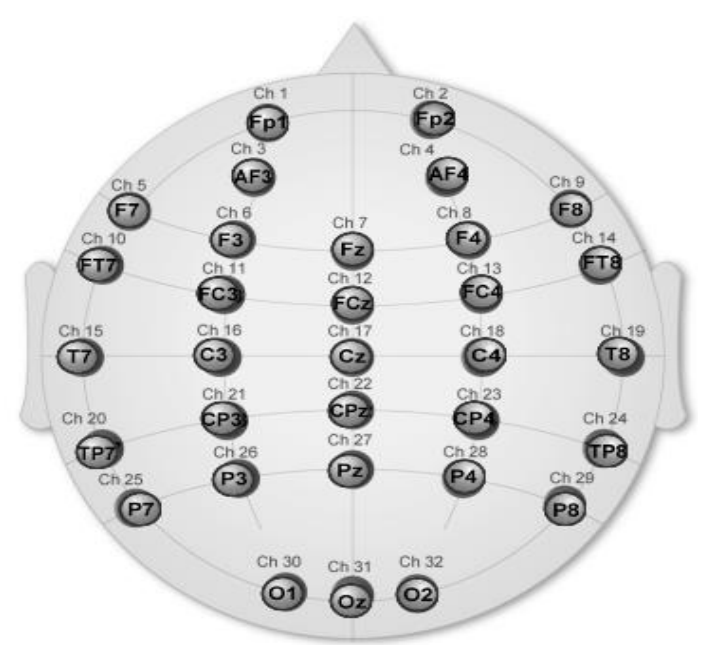

Figure 2. 32 channels of BRi32

In this study, 26 channels were used from 32 channels, Fp1, Fp2 AF3, AF4, F7, F8, FT7, FC3, FC4, FT8, T7, C3, C4, T8, TP7, CP3, CPZ, CP4, TP8, P7, P3, P4, P8, O1 and O2. The EEG raw data was sampled down to $128 \mathrm{~Hz}$ and filtered from $0.5 \mathrm{~Hz}$ to $64 \mathrm{~Hz}$ with bandpass. Then HHT was used as feature extractor to extract the feature of EEG data from post stroke patients and healthy subjects.

Figure 3 shows the time length of data acquisition consists four memorize task and four recall tasks for working memory. Each memorize and recall task was conducted for 10 seconds with 5 seconds rest between tasks. 


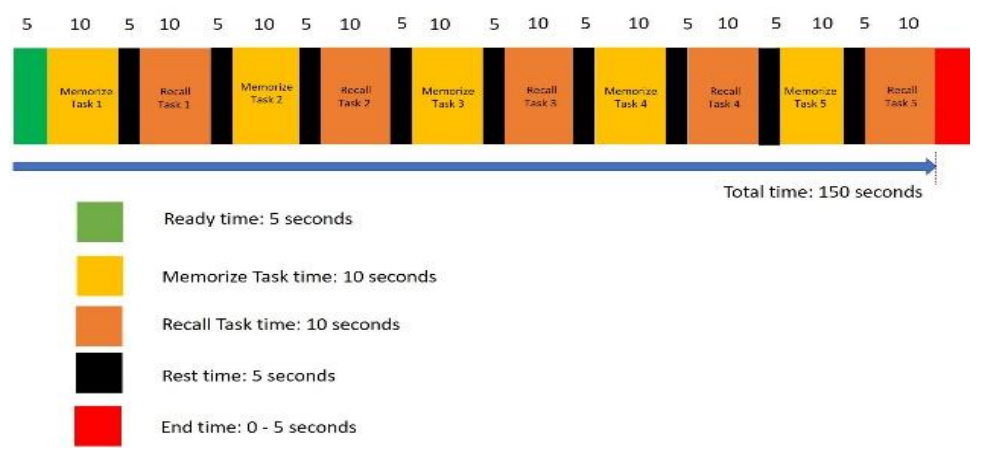

Figure 3. Data acquisition time length experiment

The subjects are asked to be motionless as possible. Five memorize task and five recall tasks appeared on the TV screen alternately for 10 seconds, the subjects were asked to rest for 5 seconds after each task. Memorize phase and recall phase was involved in the EEG data collection. EEG signal at recall phase will be used as a marker to find out the abnormalities of the EEG signal between healthy person and post stroke patient.

\subsection{Hilbert Huang Transform}

In earlier studies, spectral entropy and energy features of Hilbert Marginal Spectrum (HMS) correlate to EEG frequency for detection of seizures has been used [25].

The HHT is derived from empirical mode decomposition (EMD) and Hilbert spectral analysis [26]. EMD is decompose the data to get the intrinsic mode function (IMFs) of the signal which are amplitude and frequency modulated signal.

IMF is a data embedded in oscillation mode [26, 27]. Each element must comply with certain criteria: the mean value of the two envelopes defined respectively by local maxima and local minima must be zero and the maxima and minima value of zero-crossing must be equal or differ to one [26, 27]. Figure 4 shows how the original signal is decomposed to IMFs.

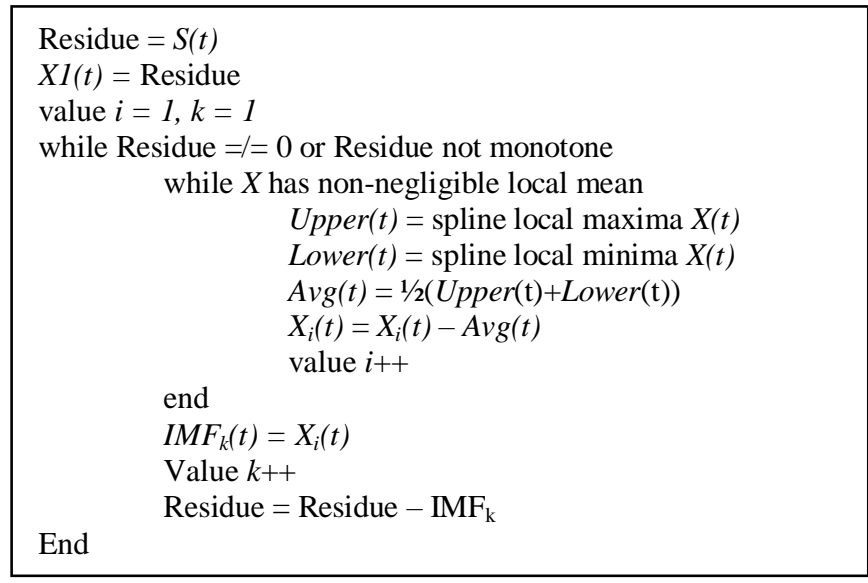

Figure 4. Pseudocode decompose original signal into IMFs

The EMD result was a decomposition of [27]:

$$
S(t)=\sum_{i}\left(I M F_{i}\right)+\text { residue }
$$

Hilbert Transform is applied to obtain IMFs can be described as:

$$
H[x(t)]=x * \frac{1}{\pi t}=y
$$


Lastly, the marginal spectrum h(w), identified by the Hilbert - Huang spectrum, would be described as:

$$
h(w)=\int_{0}^{T} H(w, t) d t
$$

From each frequency value, the marginal spectrum provides a measure of complete amplitude (or power) contribution. [26].

\section{EXPERIMENT RESULT AND DISCUSSION}

The extracted feature of EEG signal based on HHT and FFT from 43 patients of stroke and 27 subjects of healthy control were fed into ELM to get the classification performance. The parameter setting used for the experiment is shown in Table 1.

Table 1. Parameter Setting of Experiment

\begin{tabular}{cl}
\hline Technique & \multicolumn{1}{c}{ Parameter } \\
\hline HHT & Energy of Hilbert marginal spectrum \\
FFT & Power spectral density \\
ELM & Sigmoidal function \\
& Classification mode \\
& 150 hidden neurons \\
\hline
\end{tabular}

Several experiments were performed on the set of hidden neurons used in ELM and the 150 was used because it shows higher accuracy. The Table 2 express overall classification performance between HHT and FFT. Based on the Table 2, the percentage of mean testing accuracy using HHT as feature extractor was $59.14 \%$ and the percentage mean of mean testing accuracy using FFT was $94.5 \%$. As shown the mean percentage of testing accuracy using FFT is higher than using HHT as feature extractor.

Table 2. Statistic of the Testing Accuracy

\begin{tabular}{ccc}
\hline Statistic & Testing Accuracy & Testing Accuracy \\
& $(\mathrm{HHT})$ & $0.9445(94.45 \%)$ \\
Mean & $0.5914(59.14 \%)$ & 1.0000 \\
Maximum & 0.7619 & 0.7143 \\
Minimum & 0.5238 & 0.1143 \\
Standard Deviation & 0.0958 & (FFT) \\
\hline
\end{tabular}

\section{CONCLUSION}

In general, based on previous research, HHT method produces better resolution of data than FFT with regard to HHT can obtain better data in the frequency domain and time domain than FFT. In this paper, HHT was proposed as the feature extractor to classify between the $\mathrm{VaD}$ and healthy subjects. However, in this study, the result of HHT testing accuracy is lower in which 59.14\% than FFT 94.5\%. For further study, the process of signal decomposing into several IMF and selection of the IMF will be investigated to check whether this parameter effect the testing accuracy.

\section{ACKNOWLEDGEMENTS}

The authors would like to thank Universiti Teknologi Malaysia for funding this research project through a research university Grant (Q.K130000.2443.04G73) titled "CRG 8.1: AI Predictive Model for HVAC Operation".

\section{REFERENCES}

[1] Al-qazzaz NK, Ali SH, Ahmad SA., "Cognitive assessments for the early diagnosis of dementia after stroke," pp. 1743-1751, 2014

[2] Cullen B, Neill BO, Evans JJ, Coen RF, Lawlor BA., "A review of screening tests for cognitive impairment," pp. 790-799, 2007

[3] Chellappan K, Mohsin NK, Hamid S, Ali B, Islam S.," Post-stroke brain memory assessment framework. 2012, July 2014. 
[4] Erkinjuntti T, Gauthier S., "The concept of vascular cognitive impairment. Front Neurol Neurosci, "vol. 24, pp. 79-85, 2009, Available from: https://doi.org/10.1159/000197886

[5] Al-qazzaz NK, Hamid S, Ali B, Ahmad SA, Chellappan K, Islam S, et al.,"Role of EEG as biomarker in the early detection and classification of dementia, 2014.

[6] Baddeley A., "Working memory components of working memory the slave systems of working memory individual differences in working memory, pp. 255.

[7] Stinear CM, Ward NS., "How useful is imaging in predicting outcomes in stroke rehabilitation? Int j stroke," vol. 8(1), pp. 33-37, 2013, Available from: https://doi.org/10.1111/j.1747-4949.2012.00970.x

[8] Delorme A, Makeig S., "EEGLAB : An open source toolbox for analysis of single-trial EEG dynamics including independent component analysis," vol. 134, pp. 9-21, 2004.

[9] Zhang A., "A multi-view deep learning method for epileptic seizure detection using short-time fourier transform," pp. 213-22, 2017.

[10] Durongbhan P, Zhao Y, Member S, Chen L, Zis P, Marco M De, et al., "Frequency and time-frequency features based on EEG signals," IEEE Trans Neural Syst Rehabil Eng. IEEE, vol. 27(5), pp. 826-835, 2019.

[11] Neto E, Allen EA, Aurlien H, Nordby H, Eichele T., "EEG spectral features discriminate between Alzheimer's and vascular dementia, vol. 6, pp. 1-9, Feb 2015.

[12] Hossain ABMA, Rahman W, Riheen MA., "Left and right hand movements EEG signals classification using wavelet transform and probabilistic neural network," vol. 5(1), pp. 92-101, 2015.

[13] George FP, Shaikat IM, Ferdawoos PS., "Recognition of emotional states using EEG signals based on timefrequency analysis and SVM classifier," vol. 9(2), pp. 1012-1020, 2019.

[14] Yulianto E, Susanto A, Sri Widodo T, Wibowo S., "Classifying the EEG signal through stimulus of motor movement using new type of wavelet," IAES Int J Artif Intell, vol. 1, 2012.

[15] Al-Qazzaz NK, Ali SHM, Islam S, Ahmad SA, Escudero J., "EEG Wavelet Spectral Analysis During a Working Memory Tasks in Stroke-Related Mild Cognitive Impairment Patients," In: Ibrahim F, Usman J, Mohktar MS, Ahmad MY, editors. International Conference for Innovation in Biomedical Engineering and Life Sciences. Singapore: Springer Singapore, pp. 82-85, 2016.

[16] Al-qazzaz NK, Hamid S, Mohd B, Ahmad SA, Islam MS, Escudero J., "Discrimination of stroke-related mild cognitive impairment and vascular dementia using EEG signal analysis," Medical \& Biological Engineering \& Computing, pp. 137-157, 2018.

[17] Asyraf H, Shapiai MI, Setiawan NA, Musa WSNW., "Masking covariance for common spatial pattern as feature extraction, vol. 8(11), pp. 1-6, 2016.

[18] Adam A, Shapiai MI, Mohd Tumari MZ, Mohamad MS, Mubin M., "Feature selection and classifier parameters estimation for EEG signals peak detection using particle swarm optimization. sci world j," pp. 1-13, 2014, Available from: http://www.hindawi.com/journals/tswj/2014/973063/

[19] Molnár M, Osman-Sági J, Nagy Z, Kenéz J.," Scalp distribution of the dimensional complexity of the EEG and the P3 ERP component in stroke patients," Int J Psychophysiol, vol. 34(1), pp. 53-63, 1999.

[20] Barbancho MA, Berthier ML, Navas-sánchez P, Dávila G, Green-heredia C, García-alberca JM, et al." Brain \& Language Bilateral brain reorganization with memantine and constraint-induced aphasia therapy in chronic poststroke aphasia: An ERP study. Brain Lang," Elsevier Inc, pp. 145-146:1-10, 2015, Available from: http://dx.doi.org/10.1016/j.bandl.2015.04.003

[21] Coben LA, Danziger W, Storandt M.," A longitudinal EEG study of mild senile dementia of Alzheimer type: changes at 1 year and at 2.5 years," Electroencephalogr Clin Neurophysiol, vol. 61(2), pp. 101-112, 1985, Available from: http://www.sciencedirect.com/science/article/pii/001346948591048X

[22] Escudero J, Hornero R, Abásolo D, Fernández A, Escudero J, Telecomunicación ETSI De.," Blind source separation to enhance spectral and non-linear features of magnetoencephalogram recordings. Application to Alzheimer $\hat{a} €^{\mathrm{TM}} \mathrm{s}$ disease, pp. 1-28.

[23] Dressler O, Schneider G, Stockmanns G, Kochs EF.," Awareness and the EEG power spectrum: analysis of frequencies," Br J Anaesth, vol. 93(6), pp. 806-809, 2004, Available from: http://dx.doi.org/10.1093/bja/aeh270

[24] Sastra Kusuma Wijaya, C. Badri, J., "Misbach TPS and VS. Electroenchephalography (EEG) for Detecting Acute Ischemic Stroke," 2015 4th Int Conf Instrumentation, Commun Inf Technol Biomed Eng Bandung, pp. 2-3, 2015, vol. 4, pp. 42-48, 2015.

[25] Fu K, Qu J, Chai Y, Zou T., "Hilbert marginal spectrum analysis for automatic seizure detection in EEG signals. Biomed Signal Process Control," Elsevier Ltd, vol. 18, pp. 179-185, 2015, Available from: http://dx.doi.org/10.1016/j.bspc.2015.01.002

[26] Huang NE, Shen Z, Long SR, Wu MC, Shih HH, Zheng Q, et al., "The empirical mode decomposition and the Hilbert spectrum for nonlinear and non-stationary time series analysis," In: Proceedings of the Royal Society of London A: Mathematical, Physical and Engineering Sciences. The Royal Society, pp. 903-995, 1998.

[27] Huang NE, Wu Z., "A Review on Hilbert-Huang transform : Method and its applications," vol. 46, pp. 1-23, 2007, Oct 2008 Available from: http://rcada.ncu.edu.tw/reference010.pdf 


\section{BIOGRAPHIES OF AUTHORS}
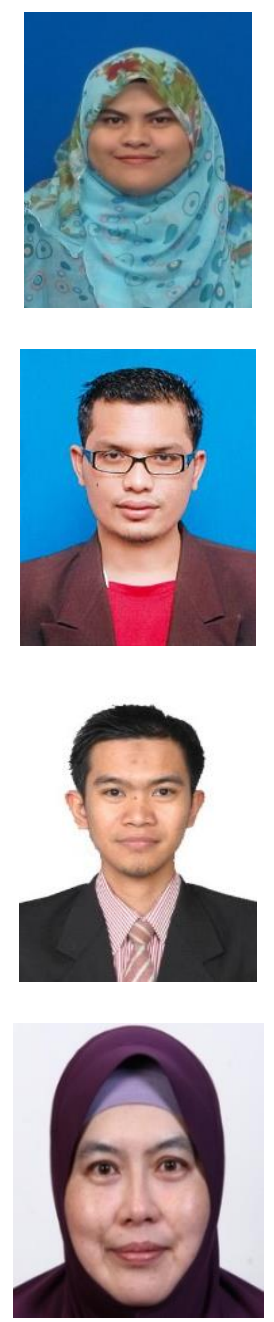

Wan Siti Nur Shafiqa Wan Musa received her Bachelor of Computer Science (Software Development) from Universiti Sultan Zainal Abidin (UniSZA) in 2015. Currently she is preparing for higher studies and research. Her research interests are biomedical signal processing.

Dr. Mohd Ibrahim Shapiai is a senior lecturer at Universiti Teknologi Malaysia. He received MEng from University of York, UK in 2007 and PhD from Universiti Teknologi Malaysia in the area of machine learning in 2013. During March until April 2010 he was a visiting researcher at Graduate School of Information, Production and Systems, Waseda University in Japan under Prof. Dr. Junzo Watada supervision and also from Jun until July 2012 at Faculty of Engineering, Leeds University, United Kingdom under Prof. Dr. Vassili Toropov supervision. His research interest are Artificial Intelligence, Machine Learning, Brain Computer Interface and Swarm Intelligence. Has been appointed as Certified NVIDIA Deep Learning Instructor.

Hilman Fauzi received his Master degree from STEI Institut Teknologi Bandung, Indonesia in 2013. He is a researcher of signal processing and lecturer at Telkom University Indonesia. Currently, he is a Ph.D candidate at Malaysia-Japan International Institute of Technology, Universiti Teknologi Malaysia (UTM), Malaysia. His research interests include signal processing and biomedical engineering.

Assoc. Prof. Dr. Aznida Firzah graduated from Faculty Of Medicine, Universiti of Malaya 1992. She served as a Medical Officer In the Ministry of Health from 1993-2003. After completing her Masters degree in Family Medicine, she joined Universiti Kebangsaan Malaysia as a lecturer and Family Medicine Specialist in May 2003, combining clinical research with teaching of both undergraduate and postgraduate students. She embarked on her PhD studies in July 2010 at the United Nations University International Institute of Global Health and is currently awaiting her viva for her work on 'The Development Of An Integrated Care Pathway For Post Stroke Patients Residing At Home In The Community: A Study Assessing Impact On Quality Of Life And Cost Effectiveness". Her current post is Associate Professor and Family Medicine Consultant in the Department of Family Medicine, Faculty of Medicine, UKM. Her area of interest includes noncommunicable diseases, chronic care, cost analysis and health systems research. Her research collaboration networking involves Dentistry, Rehabilitation and Allied Sciences and Health Economics. Her current projects include development of web- based monitoring system for longterm stroke patients at community level, working with multidisciplinary community based public health facilities in Malaysia. 